\title{
Intervención Enfermera sobre el nivel de conocimientos del paciente en cuidados postquirúrgicos
}

\section{Nurse intervention on the level of knowledge of the patient in post-surgical care}

\section{Intervenção enfermeira do nível de conhecimento no atendimento ao paciente pós-cirúrgico}

\author{
Alejandra Fuentes-Ramirez*
}

Recibido:

5 Diciembre de

\section{Para citar este artículo / To reference this article / Para citar este artigo/}

Fuentes-Ramíre A. Intervención Enfermera sobre el nivel de conocimientos del paciente en cuidados postquirúrgicos. Rev. cienc. cuidad. 2017; 14(2): 65-79.

Aprobado:

31 Mayo de 2017

\section{Resumen}

Objetivo: Evaluar una intervención enfermera sobre el nivel de conocimientos de los pacientes sobre los cuidados postquirúrgicos en un hospital de II nivel de complejidad. Materiales y métodos: Proyecto de gestión del cuidado utilizando como referente metodológico el marco lógico adaptado. Se formuló una estructura conceptual-teórico-empírica para la investigación, que artículo los conceptos de Adaptación de Roy, educación de la teoría de la incertidumbre en la enfermedad de Mishel y se formularon los indicadores empíricos a partir de los diagnósticos, clasificación de resultados y las intervenciones enfermeras para dar solución al problema de la práctica. La muestra estuvo conformada por 101 pacientes adultos, con riesgo quirúrgico I y II, que fueron intervenidos quirúrgicamente y permanecían hospitalizados. Se aplicaron como instrumentos de recolección de información previo y posterior a la intervención una valoración verbal de los sentimientos sobre la cirugía y evaluación de conocimientos sobre los cuidados posquirúrgicos. La intervención incluyó educación personalizada, proyección de videos y explicación con folletos. Resultados: El uso de la intervención permitió mejorar los resultados con relación al nivel de conocimientos en actividad, dieta, cuidados de la herida y los signos y síntomas de infección, pasando de un nivel de conocimientos inicial nulo o bajo $(1,2)$ a un nivel sustancial o extenso $(4$ o 5), según la escala de resultados. Conclusión: La intervención educativa es efectiva al mejorar el nivel de conocimientos de los pacientes con relación a los cuidados posquirúrgicos, así como los pacientes le asignan un significado positivo a la cirugía y la describen como una oportunidad. Este estudio resalta el uso de conocimiento disciplinar en la práctica de enfermería

Palabras clave: Educación en salud, enfermería, enfermería perioperatoria, teoría de enfermería.

\section{Abstract}

Objective: To evaluate a nursing intervention regarding the level of knowledge of the patients in postsurgical care in a hospital of level 2 of complexity. Materials and Methods: Project of care management using as a methodological reference the adapted logical framework, a conceptual -theoretical- empirical structure was formulated for the research that articulated the concepts of Roy's adaptation model, education of the Mishel uncertainty of illness theory and empirical indicators were formulated from the diagnoses, classification of results, and nursing interventions to resolve the problem of practice. The sample was made of 101 adult patients, with surgical risk I y II that were surgically intervened and remained hospitalized. The instruments applied for the collection of pre and post intervention were a verbal assessment of the feelings about the surgery and an evaluation of knowledge about postsurgical care. The intervention included personalized education, video projections and explanation with brochures. Results: The use of intervention allowed the results to

\footnotetext{
* Enfermera, Doctora en Enfermería. Correo: alejandra. fuentes@ unisabana.edu. co. Orcid: 00000003-3311-9211. Profesora Facultad de Enfermería y Rehabilitación, Universidad de La Sabana, Chía, Colombia.
} 
ISSN-PRINT

1794-9831

E-ISSN 2322-7028

Vol. 14 No. 2

Jul - Dic 2017

Cúcuta, Colombia

improve with relation to the level of knowledge in activity, diet, care of the wound and the signs and symptoms of infection, going from an initial level of knowledge null or low $(1,2)$ to a substantial or extensive level (4 or 5), according to this scale of results. Conclusion: The educative intervention is effective for the improvement of knowledge of the patients with relation to the postsurgical care, as well as patients that give a positive significance to the surgery and describe it as an opportunity. This study highlights the use of disciplinary knowledge in the practice of nursing.

Keywords: Health education, nursing, pre-post-operative nursing, theory of nursing.

\section{Resumo}

Objetivo: Avaliar uma intervenção de enfermagem sobre o nível de conhecimentos dos pacientes sobre os cuidados pós-cirúrgicos num hospital de II nível de complexidade. Materiais e métodos: Projeto de gestão do cuidado utilizando como referente metodológico o marco lógico adaptado. Formulou-se uma estrutura conceptual -teórico-empírica- para a pesquisa que articulou os conceitos de Adaptação de Roy, educação da teoria da incerteza na doença de Mishel e se formularam os indicadores empíricos a partir dos diagnósticos, classificação de resultados e as intervenções de enfermagem para dar solução ao problema da prática. A amostra esteve conformada por 101 pacientes adultos, com risco cirúrgico I e II, que foram operados cirurgicamente e permaneciam hospitalizados. Aplicaram-se como instrumentos de recolecção de informação antes e após à intervenção uma valoração verbal dos sentimentos sobre a cirurgia e avaliação de conhecimentos sobre os cuidados pós-cirúrgicos. A intervenção incluiu educação personalizada, projeção de vídeos e explicação com folhetos. Resultados: O uso da intervenção permitiu melhorar os resultados com relação ao nível de conhecimentos em atividade, dieta, cuidados da ferida e os signos e sintomas da infecção, passando de um nível inicial de conhecimentos nulo ou baixo $(1,2)$ a um nível substancial ou extenso (4 ou 5), segundo a escala de resultados. Conclusão: A intervenção educativa é efetiva ao melhorar o nível de conhecimentos dos pacientes com relação aos cuidados pós-cirúrgicos, assim como os pacientes lhe assignam um significado positivo à cirurgia e a descrevem como uma oportunidade. Este estudo ressalta o uso do conhecimento disciplinar na prática de enfermagem.

Palavras-chave: Educação em saúde, enfermagem, enfermagem perioperatória, teoria de enfermagem.

\section{Introducción}

Según la Organización Mundial de la Salud (1), "se calcula que en todo el mundo se realizan cada año 234 millones de operaciones de cirugía mayor, lo que equivale a una operación por cada 25 personas", de las cuales entre un $60 \%$ y $75 \%$ son ambulatorias (2) o de estancia intrahospitalaria corta, lo que implica que el tiempo de cuidado directo proporcionado por el equipo de salud disminuye y el paciente debe asumir su cuidado en casa. Por ello, la educación que la persona obtenga sobre sus cuidados postoperatorios (3) va a ser de especial importancia para su recuperación y retorno a la vida cotidiana (4).

Es así que el equipo de enfermería en cirugía debe trabajar por educar a los pacientes antes de que salgan de la institución, para que logren tener un mejor afrontamiento de la situación de salud cuando se encuentren en casa. Además, la educación para la salud debe enfocarse en "permitir a los pacientes tomar decisiones informadas que favorezcan la salud y conseguir su participación en el proceso mediante una actitud crítica y toma de decisiones que favorezcan la salud" (5), más si se tiene en cuenta que "la cirugía despierta emociones completamente opuestas, por un lado la tranquilidad y la alegría de mejorar y, por otro lado, el miedo a enfrentar el proceso quirúrgico" (6).

Este proceso educativo es liderado por el profesional de enfermería debido a que tiene una interacción constante con el paciente durante el proceso quirúrgico (6) y, más aun, cuando la medición y la notificación de los resultados de los pacientes deben ser visibles y es urgente divulgar los resultados que las enfermeras obtienen en el cuidado de los pacientes (10), entre los cuales la educación es un pilar fundamental.

Así mismo, en el área de cirugía se han detectado algunos temas específicos que deben tenerse en cuenta 
en la educación sobre cuidados relacionados con el paciente y su familia (7) (8), lo cuales se relacionan con la demostración que ellos hacen sobre las respuestas al procedimiento quirúrgico, los requisitos nutricionales, el manejo de medicamentos, el manejo del dolor, la participación en el proceso de rehabilitación y la cicatrización (9), temas que requieren ser abordados antes de que el paciente salga de la institución.

A medida que se percibe la necesidad de educación (11) nace la necesidad de indagar y consolidar resultados que respalden la importancia de la educación al paciente en los cuidados postoperatorios $(12,13)$ asumiendo que educar al paciente es un comportamiento propio de enfermería que se basa en un conocimiento disciplinar.

Con el fin de hacer explícito el uso de los marcos conceptuales, teóricos y métodos empíricos usados en esta investigación, y acogiendo la propuesta de J. Fawcett (14), se construyó una estructura conceptualteórico- empírica como guía. Específicamente, el modelo conceptual en el que se basó la investigación fue el modelo conceptual de adaptación de Callista Roy (15), la teoría de rango medio que se probó fue la teoría de incertidumbre en la enfermedad de Merle Mishel (16) y los métodos empíricos de investigación que se utilizaron fueron el plan de cuidados de enfermería a través del uso del lenguaje estandarizado, con los diagnósticos enfermeros, intervenciones y evaluación de resultados a través de la escala de resultados de enfermería (NOC) (17).

A continuación se describe cada componente de la estructura conceptual-teórico-empírica:

El componente conceptual de la estructura seleccionado fue el modelo de adaptación de Callista Roy (15). El modelo conceptual es un punto de partida para la investigación, los conceptos y proposiciones relativamente abstractas y generales que constituyen el modelo conceptual no pueden ser directamente probadas. La función del componente conceptual de la estructura es guiar la investigación, proporcionando un marco distintivo, de referencia, o un amplio lente a través del cual se ve la investigación (14).

El modelo de Adaptación de Roy actúa como un paraguas para la teoría y métodos de investigación de la estructura, provee un horizonte y una meta en la investigación que, para este caso, se asume como la adaptación de los pacientes a su estado de salud posquirúrgico, quienes están en un ambiente directamente relacionado con los estímulos. Roy define los estímulos como todo aquello que provoca una respuesta; por eso, para este trabajo, se definió el estímulo como esas nuevas situaciones que debe asumir la persona para cuidarse, teniendo en cuenta el proceso quirúrgico que está viviendo.

Se utilizó el componente teórico porque las teorías son más reducidas en su alcance que un modelo conceptual, pero más cercanas a lo que se observa en la cotidianidad y en la práctica profesional (14). Para la teoría de la incertidumbre en la enfermedad "los pacientes procesan cognitivamente los estímulos relacionados con la enfermedad y construyen un significado en estos eventos. La incertidumbre o la incapacidad para estructurar significado puede desarrollarse si el paciente no forma un esquema cognitivo para los eventos de la enfermedad" (16). Manejar la incertidumbre asociada con una enfermedad y su tratamiento puede ser una tarea esencial en la adaptación.

Mishel, en la teoría de la incertidumbre, identifica tres variables que la preceden: "el marco de estímulos, la capacidad cognitiva y los proveedores de la estructura, los cuales ofrecen la información que es procesada por el paciente". Este trabajo se basa en la segunda variable, que influye sobre el marco de estímulos y los proveedores de estructura -los cuales son "los recursos disponibles para ayudar a la persona en la interpretación del marco de estímulos. Se ha propuesto que los proveedores de estructura reducen el estado de incertidumbre tanto directa como indirectamente" (16).

Así mismo, Mishel describe que "la reducción de la incertidumbre ocurre indirectamente cuando los proveedores de estructura ayudan al paciente a determinar el patrón de los síntomas, la familiaridad con el evento y la congruencia de experiencias. Los proveedores de estructura son autoridades confiables por su nivel educativo y apoyo social" (16), que, para este caso, se asumen como los profesionales de enfermería que realizan el proceso educativo.

Mishel define que la educación es un componente de los proveedores de estructura que "puede ayudar a suplir 
ISSN-PRINT

$1794-9831$

E-ISSN 2322-7028

Vol. 14 No. 2

Jul - Dic 2017

Cúcuta, Colombia la estructura de los eventos en el marco de estímulos, incrementando la base de conocimientos con los cuales se asocian estos eventos, dándoles así significado y contexto" (16). Esto implica que la educación es un punto de alta importancia para esta población.

Cuando una persona va a ser sometida a una intervención quirúrgica, si no recibe educación sobre su proceso sus niveles de incertidumbre pueden aumentar. La teoría de la incertidumbre propone que la educación es un recurso disponible que reduce el estado de incertidumbre tanto directa como indirectamente. Por esta razón, la educación del paciente es fundamental para la recuperación postoperatoria (18) y se ha convertido en un aspecto importante de la atención de enfermería en los últimos años (19). Con relación a la incertidumbre, está demostrado cómo los pacientes, con una intervención educativa adecuada, la reducen sustancialmente $(2,20,21)$.

El último componente de la estructura está relacionado con los métodos empíricos de investigación, que integran la parte más concreta y específica de la investigación. Este componente permite identificar las formas de obtener la información y provee las técnicas analíticas necesarias para llegar a las conclusiones sobre la prueba de la teoría (14). Para este caso, en la literatura está reportada la responsabilidad de la enfermera en la consecución de planes de cuidado que promuevan la prevención de complicaciones postoperatorias (22), entre las cuales se encuentran los cuidados relacionados con la actividad física (23), la dieta, el control de la infección y los cuidados de la herida postoperatoria. De igual forma, la ejecución y evaluación de las actividades propias de educación en la práctica de enfermería (24) son esenciales para validar el rol de las enfermeras en escenarios como el quirúrgico.

Teniendo en cuenta lo anterior, se consideró la importancia de investigar en el área de educación al paciente quirúrgico, operacionalizando los conceptos teóricos de la disciplina así como sus aportes a nivel social, teórico y disciplinar. De tal forma que, como lo señala Morales-Aguilar y Col, para fortalecer la disciplina, se requiere "investigación acerca de la aplicación de componentes teóricos y metodológicos sobre los cuidados de enfermería, ya que la aplicación está restringida por su complejidad y difícil comprensión por parte del personal que trabaja en las instituciones de salud" (25). Por lo tanto, al usar la teoría en la práctica se cumple con el objetivo de aportar a la disciplina y construir aportes teóricos que ejemplaricen el uso en la práctica

El uso de las teorías permite tener un "pensamiento teórico que guía la práctica a partir del razonamiento, el saber, el conocer y el evaluar y el redefinir; dan sentido al hacer y crean una estructura y una substancia que finalmente da visibilidad a la práctica" (26). Por ello, la articulación que se hace del modelo conceptual, la teoría de rango medio y los métodos empíricos de la investigación permiten concretar espacios para la aplicación de la teoría en la práctica, reafirmando el saber propio y demostrando resultados de cuidado.

Por otra parte, trasladar la teoría a la práctica permite aportar en la solución de problemas de la población -como en el caso de la necesidad de educación a los pacientes posquirúrgicos-, debido a que, aunque se cuenta con programas de seguimiento postoperatorio (27), no todas las instituciones los tienen y, por lo tanto, requieren la educación necesaria para realizar su cuidado en casa.

Así mismo, esta reportado cómo la cirugía y las complicaciones derivadas de la herida tienen implicaciones en la calidad de vida de las personas, tanto en su imagen corporal, como en sus posibles alteraciones de la cotidianidad (28), así que, al enfocarse de manera individual y permitir que la persona exprese sus sentimientos y conozca cómo cuidarse, se dará un paso que tendrá implicaciones en otros resultados de cuidado como la detección y manejo en complicaciones postoperatorias (29).

Igualmente, tendrá repercusiones indirectas en la calidad de la atención del servicio de cirugía, debido a que, como lo demuestran otros estudios, la percepción de los usuarios mejora cuando la enfermera comparte mayor tiempo con ellos $(30,31)$.

\section{Materiales y métodos}

Este proyecto de gestión del cuidado utilizó la metodología del marco lógico modificado como estrategia administrativa, el cual se aplica "en servicios de salud u otros escenarios de la práctica, previa identificación de problemas prioritarios de cuidado de las personas con alternativas de solución desde la perspectiva teórica de la enfermería"(32). 
Se seleccionó esta metodología porque "la práctica de enfermería centrada en teoría de rango medio favorece el logro de las metas propuestas en beneficio de la salud de las personas y de la calidad en la prestación de los servicios de salud" (32).

Los criterios de inclusión fueron: personas adultas, clasificadas con riesgo quirúrgico I y II según la American Society of Anesthesiology (36), intervenidas quirúrgicamente en un hospital de segundo nivel de Bogotá, Colombia y que se encontraran hospitalizadas. Se excluyeron los pacientes con déficit cognitivo, con una situación de salud que limitara la adquisición de conocimientos o la comunicación, y quienes requerían hospitalización en unidades de cuidado intensivo.

El muestreo fue no probabilístico, por conveniencia, debido a que, como lo señala Burns y Grove, se "incluyeron los pacientes que fueron atendidos en unos días específicos, hospitalizados con diagnósticos médicos específicos o problemas de enfermería. En este caso, el investigador sencillamente introduce en el estudio a los sujetos disponibles" (37) que, para este caso, fueron las personas que cumplían con los criterios de inclusión y exclusión y estaban en el horario de la mañana, durante un periodo de tres meses.

La muestra final incluyó 101 participantes, 43 mujeres y 68 hombres y la edad promedio fue de 42 años (ver Figura 1).

La intervención se desarrolló en un hospital público que presta atención, en su mayoría, a personas del régimen subsidiado en salud y cuenta con servicios de atención de urgencias, hospitalización, cirugía y unidades de cuidado intensivo.

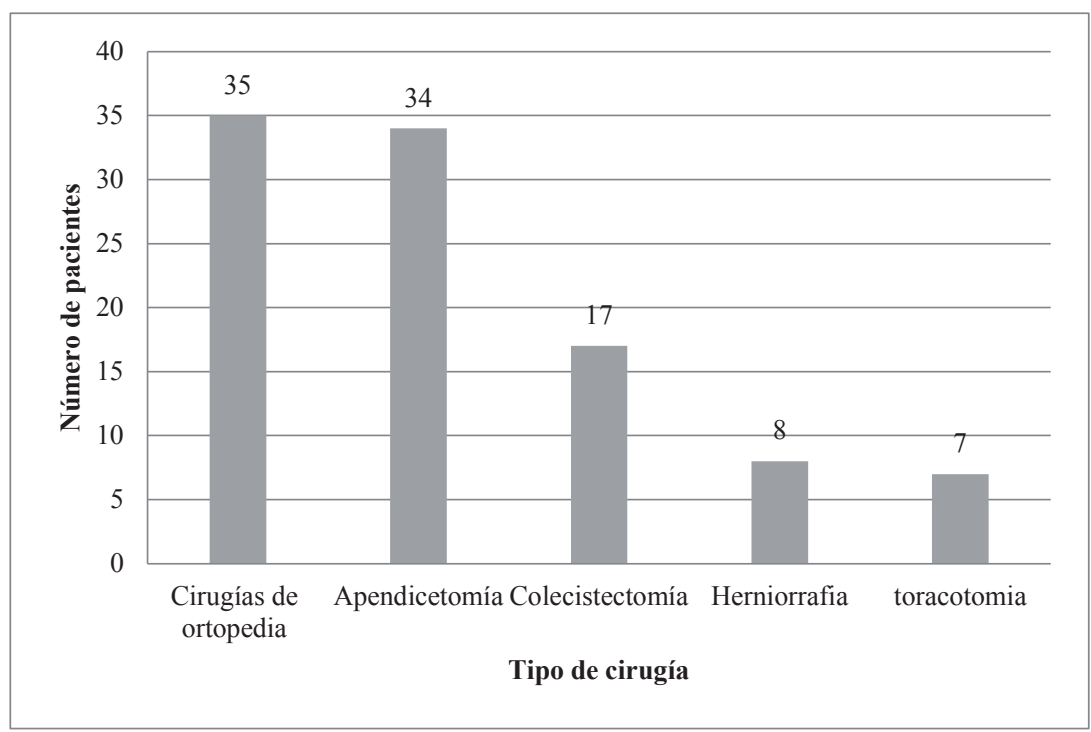

Figura 1. Número de pacientes por tipo de cirugía. n:101

Fuente: Ficha de datos sociodemográficos, 2016.

Para desarrollar la metodología del marco lógico se acogió el siguiente proceso: 1) se identificó la necesidad de educación de los pacientes del servicio de hospitalización cirugía a través de un árbol de problemas; 2) se construyó una estructura conceptualteórico- empírica para la investigación, que artículo el concepto de Adaptación del Modelo de Callista Roy y el de educación que hace parte del componente de proveedores de estructura de la teoría de incertidumbre
ISSN-PRINT

1794-9831

E-ISSN 2322-7028

Vol. 14 No. 2

Jul - Dic 2017

Cúcuta, Colombia de la enfermedad de Merle Mishel; 3) mediante el análisis de la situación, se identificó y planeó un cuidado bajo la etiqueta diagnóstica de enfermería NANDA (33) Conocimientos Deficientes, se estableció la meta con indicadores verificables que se adaptaron teniendo en cuenta la estructura NOC (17) y se realizaron las intervenciones según lo planteado en las Intervenciones de Enfermería (NIC) (34), según sus siglas en inglés, cómo se muestra en la Tabla 1: 
ISSN-PRINT

1794-9831

E-ISSN 2322-7028

Vol. 14 No. 2

Jul - Dic 2017

Cúcuta, Colombia

Tabla 1. Aplicación de la estructura conceptual teórico empírico para el desarrollo de la intervención.

\begin{tabular}{|c|c|c|c|}
\hline & Componente seleccionado & Premisa & Aplicación con lenguaje estandarizado \\
\hline Conceptual & $\begin{array}{l}\text { Modelo conceptual: } \\
\text { Adaptación Callista Roy }\end{array}$ & $\begin{array}{l}\text { Promover la adaptación de las perso- } \\
\text { nas a la cirugía. }\end{array}$ & $\begin{array}{l}\text { Diagnóstico de enfermería. (NANDA): } \\
\text { Conocimientos deficientes relacionados } \\
\text { con las nuevas situaciones que debe asumir } \\
\text { la persona para cuidarse en casa debido el } \\
\text { proceso quirúrgico que está viviendo }\end{array}$ \\
\hline Teórico & $\begin{array}{l}\text { Teoría de la Incertidumbre } \\
\text { en la enfermedad de Merle } \\
\text { Mishel. }\end{array}$ & $\begin{array}{l}\text { La educación mejora la adaptación. } \\
\text { La educación es un recurso disponible } \\
\text { que reduce el estado de incertidumbre, } \\
\text { tanto directa como indirectamente. }\end{array}$ & $\begin{array}{l}\text { Meta o resultados de enfermería (NOC): } \\
\text { educación sobre } \\
\text { - Actividad prescrita } \\
\text { - Dieta prescrita } \\
\text { - Control de la infección } \\
\text { - Curación de la herida }\end{array}$ \\
\hline Empírico & $\begin{array}{l}\text { Valoración, Protocolos de } \\
\text { intervención. }\end{array}$ & $\begin{array}{l}\text { Al realizar la valoración se conocen } \\
\text { las vivencias de la persona. } \\
\text { Enseñanza sobre los cuidados posqui- } \\
\text { rúrgicos. }\end{array}$ & $\begin{array}{l}\text { Intervenciones de enfermería (NIC): } \\
\text { - Enseñanza ejercicio prescrito } \\
\text { - Enseñanza dieta prescrita } \\
\text { - control de infecciones } \\
\text { - Cuidados del sitio de la incisión }\end{array}$ \\
\hline
\end{tabular}

Fuente: Adaptado de Fawcett y DeSanto (2013). Moorhead S, Johnson M, Maas M, Swanson (2013). (35,36)

Para recolectar la información se utilizaron los siguientes instrumentos:

a) Una entrevista semiestructurada que indagó sobre los sentimientos que tenía la persona sobre la cirugía.

b) Se desarrolló una ficha de datos sociodemográficos en la que se consignó edad, género, tipo de cirugía.

c) La Escala de evaluación NOC, donde se definieron los parámetros según la Clasificación de Resultados de Enfermería (NOC) (17), por sus siglas en inglés, la cual es una clasificación completa y normalizada de los resultados del paciente, desarrollada para evaluar los efectos de las intervenciones previstas por las enfermeras $\mathrm{u}$ otros profesionales de la salud. Se tomaron los indicadores que están en la primera columna de la Tabla 2 y se especificaron para el estado esperado del paciente. Para la operacionalización de los indicadores se tuvo en cuenta lo planteado por Moorhead y Col, quienes precisan "que es muy importante cuando la enfermera cuantifica resultados. Requiere el mismo juicio utilizado al evaluar si el paciente ha satisfecho o no un objetivo, ha mejorado con relación a un objetivo o se ha deteriorado con relación a un objetivo. Algunas organizaciones han escogido proporcionar unidades de medida específicas para los resultados" (17), por lo cual en este proyecto se definen aspectos específicos para cada indicador, tal como se describe en la Tabla 2. 
Tabla 2. Operacionalización de los indicadores de evaluación de Resultados de Enfermería (NOC).

\begin{tabular}{|c|c|c|c|c|c|c|}
\hline \multirow{2}{*}{ NOC } & Indicador & 1 & 2 & 3 & 4 & 5 \\
\hline & $\begin{array}{c}\text { Definición } \\
\text { de escala }\end{array}$ & $\begin{array}{c}\text { Ningún } \\
\text { Conocimiento }\end{array}$ & $\begin{array}{l}\text { Conocimiento } \\
\text { escaso }\end{array}$ & $\begin{array}{l}\text { Conocimiento } \\
\text { moderado }\end{array}$ & $\begin{array}{l}\text { Conocimiento } \\
\text { sustancial }\end{array}$ & $\begin{array}{l}\text { Conocimiento } \\
\text { extenso }\end{array}$ \\
\hline $\begin{array}{l}\text { Conocimiento: } \\
\text { Actividad prescrita }\end{array}$ & $\begin{array}{c}\text { Actividad } \\
\text { prescrita }\end{array}$ & $\begin{array}{l}\text { No identifica } \\
\text { ningún cuidado } \\
\text { en la actividad }\end{array}$ & $\begin{array}{l}\text { Identifica un } \\
\text { cuidado en } \\
\text { la forma de } \\
\text { movilizarse }\end{array}$ & $\begin{array}{l}\text { Identifica dos } \\
\text { cuidado en } \\
\text { la forma de } \\
\text { movilizarse }\end{array}$ & $\begin{array}{l}\text { Identifica tres } \\
\text { cuidado en } \\
\text { la forma de } \\
\text { movilizarse }\end{array}$ & $\begin{array}{c}\text { Identifica cuatro } \\
\text { cuidados en } \\
\text { la forma de } \\
\text { movilizarse }\end{array}$ \\
\hline $\begin{array}{c}\text { Conocimiento: Dieta } \\
\text { prescrita }\end{array}$ & $\begin{array}{c}\text { Dieta } \\
\text { prescrita }\end{array}$ & $\begin{array}{l}\text { No identifica } \\
\text { los alimentos } \\
\text { requeridos para } \\
\text { su dieta }\end{array}$ & $\begin{array}{l}\text { Lista menos de } \\
\text { cinco alimentos } \\
\text { requeridos según } \\
\text { la dieta prescrita }\end{array}$ & $\begin{array}{l}\text { Lista entre } 6 \text { y } \\
10 \text { alimentos } \\
\text { requeridos, según } \\
\text { la dieta prescrita. }\end{array}$ & $\begin{array}{l}\text { Lista entre } 10 \text { o } \\
\text { más alimentos } \\
\text { requeridos según } \\
\text { la dieta prescrita } \\
\text { y da una razón de } \\
\text { su prescripción }\end{array}$ & $\begin{array}{l}\text { Lista entre } 10 \text { o } \\
\text { más alimentos } \\
\text { requeridos según } \\
\text { la dieta prescrita } \\
\text { y da más de } \\
\text { una razón de su } \\
\text { prescripción }\end{array}$ \\
\hline $\begin{array}{l}\text { Conocimiento: } \\
\text { Control de la } \\
\text { infección }\end{array}$ & $\begin{array}{c}\text { Signos y } \\
\text { síntomas de } \\
\text { la infección }\end{array}$ & $\begin{array}{l}\text { No identifica } \\
\text { ningún signo de } \\
\text { infección }\end{array}$ & $\begin{array}{l}\text { Lista uno a dos } \\
\text { signo de infección }\end{array}$ & $\begin{array}{c}\text { Lista de } 3 \text { a } \\
4 \text { signos de } \\
\text { infección }\end{array}$ & $\begin{array}{c}\text { Lista de } 5 \text { a } \\
6 \text { signos de } \\
\text { infección }\end{array}$ & $\begin{array}{l}\text { Lista más de } \\
6 \text { signos de } \\
\text { infección }\end{array}$ \\
\hline $\begin{array}{l}\text { Conocimiento: } \\
\text { Cuidados de la herida }\end{array}$ & $\begin{array}{l}\text { Cuidados de } \\
\text { la herida }\end{array}$ & $\begin{array}{c}\text { No conoce la } \\
\text { forma de cuidar la } \\
\text { herida }\end{array}$ & $\begin{array}{c}\text { Nombra un } \\
\text { cuidado que } \\
\text { debe tener con la } \\
\text { herida }\end{array}$ & $\begin{array}{c}\text { Nombra dos } \\
\text { cuidados que } \\
\text { debe tener con la } \\
\text { herida }\end{array}$ & $\begin{array}{c}\text { Nombra tres } \\
\text { cuidados que } \\
\text { debe tener con la } \\
\text { herida }\end{array}$ & $\begin{array}{c}\text { Nombra cuatro } \\
\text { cuidados que } \\
\text { debe tener con la } \\
\text { herida }\end{array}$ \\
\hline
\end{tabular}

Fuente: Fuentes-Ramírez A. Adaptación Escala NOC de los conocimientos posquirúrgicos, 2016
ISSN-PRINT

1794-9831

E-ISSN 2322-7028

Vol. 14 No. 2

Jul - Dic 2017

Cúcuta, Colombia
La intervención tuvo en cuenta los siguientes pasos:

1. Valoración verbal de los sentimientos que tenía la persona sobre la cirugía y la evaluación según la escala NOC de los conocimientos posquirúrgicos.

2. Ejecución de la intervención donde se tuvo como base la educación sobre el ejercicio prescrito, la dieta, el control de infecciones y cuidados de la herida, para lo cual se desarrollaron videos explicativos específicos para cada tipo de cirugía y, además, se entregaron folletos informativos sobre cada tipo de cirugía y se realizó explicación personalizada de cada cuidado. Cada sesión duró aproximadamente 30 minutos.

3. El día siguiente a la intervención se realizó la valoración verbal y evaluación como en la primera fase.

La Figura 2 resume el desarrollo de la intervención.

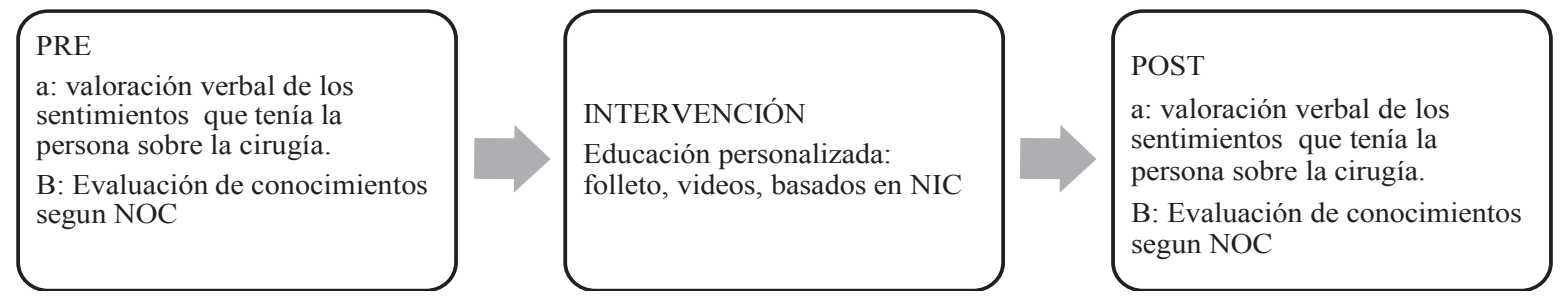

Figura 2. Etapas de la intervención.

Fuente: datos del estudio

Esta investigación tuvo en cuenta las normas nacionales e internacionales de ética, específicamente lo consagrado el código de Núremberg (38), la declaración de Helsinki (39) y la Resolución
No. 8430 de 1993 del Ministerio de Salud (40), especialmente en el Capítulo I, con los artículo 5, $6,8,10,13,14$ y 15 . En este proyecto prevaleció el respeto a la dignidad de los participantes y la 
ISSN-PRINT

1794-9831

E-ISSN 2322-7028

Vol. 14 No. 2

Jul - Dic 2017

Cúcuta, Colombia protección de sus derechos y su bienestar. Por esta razón, los pacientes, previo al inicio de la intervención, fueron informados sobre el proyecto, decidieron de manera voluntaria su participación y firmaron el consentimiento. Igualmente, se explicó la posibilidad de retirarse en cualquier momento, sin perjuicio de sufrir alguna pérdida en su tratamiento o trato con la institución de salud. Además, la información obtenida fue confidencial y sólo se manejó con fines académicos.

Se tuvieron en cuenta los principios éticos de beneficencia, entendida como la obligación de maximizar los beneficios, por lo cual a todas las personas que se encontraban en el servicio durante el periodo de aplicación del estudio y quisieron participar, se les realizó la intervención, pero sólo se incluyeron en la muestra los participantes que cumplían con los criterios de inclusión.

Con relación al principio de no maleficencia, se dejó en claro que en ningún momento se tuvo la intención de dañar o lesionar a las personas participantes. También se aplicó el principio de justicia, que considera la distribución equitativa de los riesgos y beneficios. Para la realización de este proyecto se contó con la autorización de la coordinación de cirugía y el líder del proceso de enfermería y se respetaron las políticas y normas de la institución.

\section{Objetivos}

\section{Objetivo general}

Evaluar una intervención enfermera sobre el nivel de conocimientos de los pacientes sobre los cuidados postquirúrgicos en un hospital de II nivel de complejidad.

\section{Objetivos específicos:}

- Describir los sentimientos que tiene la persona sobre la cirugía previa y posterior a la realización de la intervención.

- Medir el nivel de conocimientos de los pacientes sobre los cuidados postquirúrgicos previo a la realización de la intervención.

- Medir el nivel de conocimientos de los pacientes sobre los cuidados postquirúrgicos posterior a la realización de la intervención

- Comparar el nivel de conocimientos de los pacientes sobre los cuidados postquirúrgicos previo y posterior a la intervención.

\section{Resultados}

El proyecto se realizó durante los meses de noviembre 2015 y abril y mayo 2016. A continuación se describen los resultados obtenidos con relación a los sentimientos que refirieron los participantes y las mediciones con relación al nivel de conocimientos.

\section{Sentimientos que tiene la persona sobre la cirugía, previo y posterior a la realización de la intervención.}

Con relación a los datos obtenidos en la entrevista que valoró los sentimientos de las personas pre y post intervención, a continuación se describen los principales hallazgos obtenidos en la entrevista en los dos momentos:

a) Previo a la intervención educativa, las personas expresaban sentimientos de temor, miedo y no lograban definir con exactitud lo que significaba la cirugía. Durante la entrevista expresaban sus sentimientos con relación a las dudas que tenían sobre su recuperación, los cuidados en casa y los problemas administrativos que deberían resolver para poder salir. También refirieron dudas sobre la posibilidad de recibir visitas o consumir determinados tipos de alimentos. Además, expresaron dudas con respecto a la movilidad $\mathrm{y}$ al cuidado que deberían tener para evitar complicaciones posteriores.

b) Luego de la intervención, sus expresiones cambiaban y veían la cirugía más como una oportunidad y no como una amenaza o peligro. Adicionalmente, tenían herramientas que les posibilitaba cuidarse de una mejor forma. Con relación a cada uno de los puntos que incluía la intervención, los pacientes expresaron sus creencias respecto a los alimentos que, según ellos, podrían atrasar o tener un efecto negativo sobre la herida. Con relación al movimiento y a los cuidados de la herida, los pacientes estuvieron muy atentos y consideraban que deberían aplicar los conocimientos adquiridos debido a que el movimiento se limitaba por temor a que la herida 
se abriera o sufriera algún daño, teniendo en cuenta que la mayoría de las heridas se encontraban en la región abdominal.

Nivel de conocimientos de los pacientes sobre los cuidados postquirúrgicos previo y posterior a la realización de la intervención

a) En la evaluación de conocimientos previa a la realización de la intervención, los puntajes fueron muy bajos, según la escala NOC y oscilaron entre 1 y 2 , nulo o escaso, respectivamente, es decir, las personas no identifican ningún cuidado o solamente un cuidado con relación a la actividad prescrita, dieta, control de la infección y cuidados de la herida postoperatoria.

b) Las personas, luego de la intervención, mejoraron sus conocimientos con relación a los cuidados posquirúrgicos, ubicándose entre $4 \mathrm{y}$ 5 , es decir, conocimiento sustancial o extenso.

Los indicadores actividad prescrita y cuidados de la herida obtuvieron la mayor puntuación post intervención.

En la Figura 3 se observa la comparación entre el cambio en el nivel de conocimientos según la escala NOC previo y posterior a la intervención educativa.

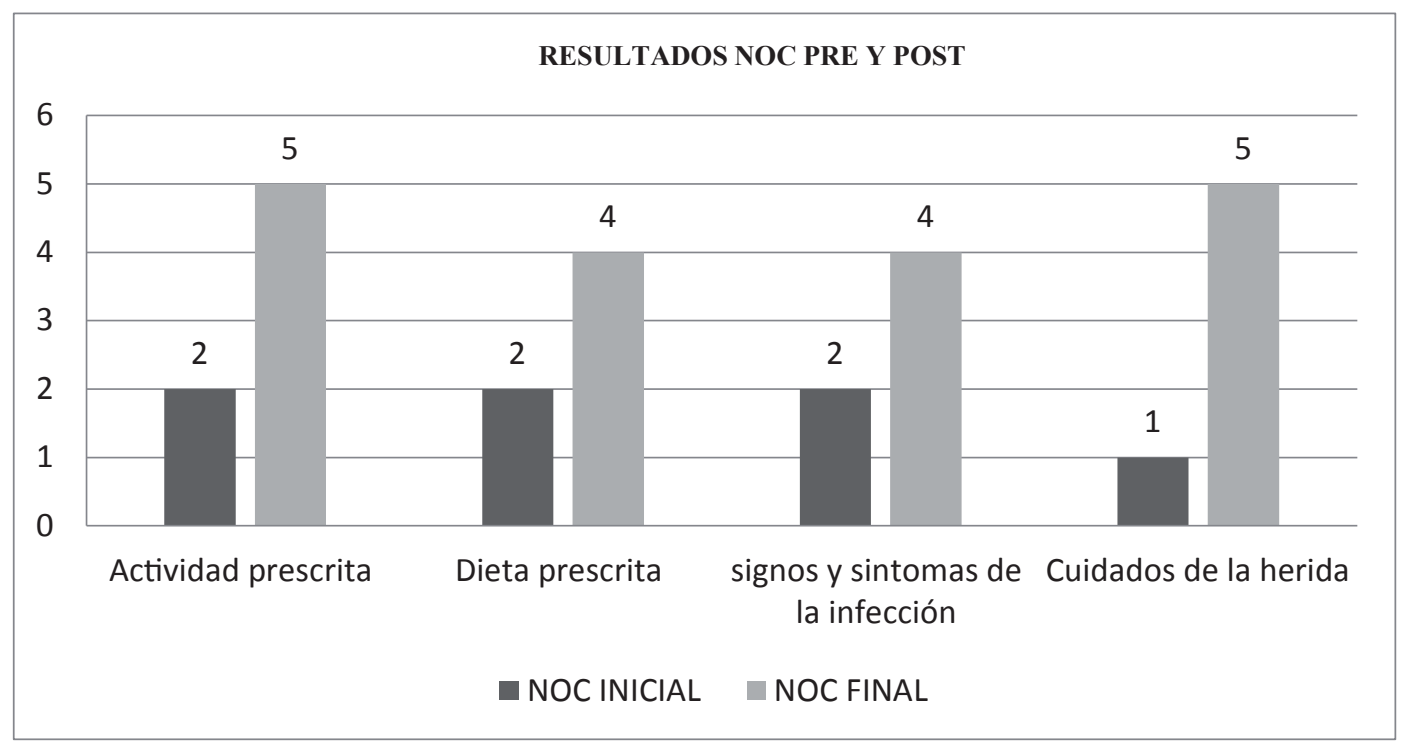

Figura 3. Cambio en el resultado previo y posterior a la intervención.

Fuente: Escala NOC de los conocimientos posquirúrgicos, 2016

\section{Análisis de Resultados Pre y Post Intervenciones de enfermería de las variables dependientes del estudio.}

Una vez obtenidos los resultados de las pruebas de normalidad de cada variable central en estudio, se procedió a definir los datos estadísticos con los cuáles se realizarían los respectivos análisis inferenciales para determinar la efectividad de la intervención enfermera sobre el nivel de conocimientos de los pacientes en los cuidados postquirúrgicos. Los resultados de estos análisis, realizados en el programa SPSS Statistics 24, se presentan a continuación, en la Tabla 3: 
ISSN-PRINT

$1794-9831$

E-ISSN 2322-7028

Vol. 14 No. 2

Jul - Dic 2017

Cúcuta, Colombia

Tabla 3: Resultados de la intervención de enfermería sobre las variables dependientes del estudio (comparación entre medidas Pre y Post)

\begin{tabular}{|c|c|c|c|c|c|c|c|c|}
\hline \multicolumn{4}{|c|}{$\begin{array}{l}\text { Resultado pre intervención } \\
\text { (n: 101) }\end{array}$} & \multicolumn{4}{|c|}{$\begin{array}{l}\text { Resultado post intervención } \\
\text { (n: 101) }\end{array}$} & \multirow[b]{2}{*}{ P valo } \\
\hline $\begin{array}{c}\text { Variable: } \\
\text { conocimiento } \\
\text { sobre }\end{array}$ & Media & $\begin{array}{l}\text { Desviación } \\
\text { estándar }\end{array}$ & Mediana & $\begin{array}{c}\text { Variable } \\
\text { conocimiento } \\
\text { sobre }\end{array}$ & Media & $\begin{array}{l}\text { Desviación } \\
\text { estándar }\end{array}$ & Mediana & \\
\hline Actividad pre & 1,87 & 0.337 & 2 & Actividad post & 4.14 & 0.347 & 4 & $0.000^{*}$ \\
\hline Dieta Pre & 1.67 & 0.471 & 2 & Dieta Post & 4.28 & 0.776 & 4 & $0.000^{*}$ \\
\hline $\begin{array}{l}\text { Signos y } \\
\text { síntomas pre }\end{array}$ & 1.65 & 0.478 & 2 & $\begin{array}{c}\text { Signos y } \\
\text { síntomas post }\end{array}$ & 4.42 & 0.495 & 4 & $0.000^{*}$ \\
\hline $\begin{array}{c}\text { Cuidados heridas } \\
\text { pre }\end{array}$ & 1.30 & 0.460 & 1 & $\begin{array}{c}\text { Cuidados heridas } \\
\text { post }\end{array}$ & 4.65 & 0.480 & 5 & $0.000 *$ \\
\hline
\end{tabular}

Prueba de t de Student para muestras emparejadas (prueba paramétrica)

Fuente: Datos del estudio

A partir de los resultados obtenidos se puede concluir que el nivel de conocimiento (medido con la escala NOC) mejoró significativamente tras la aplicación de la intervención. Los valores obtenidos para la escala NOC de conocimiento sobre actividad pre intervención fueron de $1,87 \pm .337$ y NOC Post de $4.14 \pm .347$, mostrando un cambio de dos puntos, pasando de conocimiento escaso a conocimiento sustancial.

Resultados similares se obtuvieron para el conocimiento sobre dieta, en el cual se obtuvieron datos pre de 1,67 \pm .471 , en donde se evidencia una mejoría estadísticamente significativa después de la intervención, para la medición post $4.28 \pm .776$. Para el conocimiento de signos y síntomas de infección se obtuvieron datos pre $1.65 \pm .478$ y post $4.42 \pm$ .495 , siendo más sobresaliente el cambio para el conocimiento sobre heridas, teniendo un puntaje pre $1.30 \pm .460$ comparado con el NOC post de $4.65 \pm$ $.480(\mathrm{p}=0.000)$.

En conclusión, se puede afirmar que la intervención fue eficaz y contribuyó significativamente a mejorar los conocimientos sobre el cuidado del paciente en el periodo postoperatorio.

El estudio tuvo la limitación de abordar sólo cuatro parámetros en la educación: actividad prescrita, dieta prescrita, signos y síntomas de la infección y cuidados de la herida. Se evidenció que para futuros proyectos es necesario incluir otros parámetros sobre cómo retomar la cotidianidad así como otros resultados de cuidado susceptibles de ser medidos, como las complicaciones postoperatorias, los reingresos y el acompañamiento y guía sobre los trámites administrativos y requerimientos sobre salida del hospital.

\section{Discusión}

La discusión se realizará teniendo en cuenta dos miradas: primero, los resultados obtenidos en la intervención y dos la implicación de la aplicación de la teoría de enfermería en la práctica.

Con relación a la primera mirada, se analizó que los sentimientos que tienen las personas con relación a la cirugía cambian, pues se evidenció que al trabajar en el concepto de proveedores de estructura, con el componente de educación y contar con profesionales de enfermería, permitió modelar los sentimientos de los participantes con relación a la cirugía y la forma de cuidarse en casa, teniendo presente el estímulo que significa las nuevas situaciones que debe asumir la persona para cuidarse en casa debido el proceso quirúrgico que está viviendo

Además, con relación a la intervención y su aplicabilidad en el escenario actual, existe evidencia sobre la importancia de la cirugía ambulatoria en la reducción de costos económicos para los hospitales en diferentes tipos de cirugía (41). También es evidente la necesidad de continuar realizando intervenciones que involucren y empoderen al paciente en su cuidado, con el fin de prevenir complicaciones (42) 
en el postoperatorio inmediato (43) y mediato (44). Asimismo, mantener un equipo de enfermería que pueda aportar en la construcción de un mejor cuidado para ellos (45).

Este estudio permite probar que los resultados que las intervenciones de enfermería tienen sobre el paciente son sensibles de ser medidos y pueden influir en resultados del cuidado, específicamente en el conocimiento que obtiene el paciente sobre la forma de cuidarse, así como sobre la incertidumbre y la adaptación a su nueva condición de salud, además de otros resultados que debido a las limitantes del estudio no se lograron evidenciar, tales como la ausencia de complicaciones, los resultados clínicos y los resultados de salud funcionales.

Así mismo, usar los indicadores de resultado NOC permite evaluar la eficacia de las intervenciones enfermeras y la evolución de las funciones de la enfermera en términos de la salud y conocimientos previos del paciente en ambientes de rápida interacción (46) como la cirugía ambulatoria. El uso del lenguaje estandarizado permite hacer visible el uso del conocimiento disciplinar sobre todo cuando es apoyado por teorías de enfermería. Identificar los diagnósticos prioritarios y trabajar sobre este tema es un reto que enfermería debe seguir asumiendo para su trabajo constante en el área perioperatoria (47).

El segundo aspecto, que implica la aplicación del sistema teórico empírico a la práctica como norte y guía para el desarrollo y articulación con el lenguaje estandarizado, permite, como lo plantea Duran (48), hacer uso de la teoría de enfermería en la práctica y, por tanto, aporta a cambiar "la idea de generación del conocimiento académico por la del conocimiento basado en la práctica. Esta propuesta genera una transformación del saber hacer diario de la práctica de enfermería al conocer de ese hacer, pero a través del conocimiento científico. El conocimiento científico, entonces, se convierte en el soporte central de la práctica diaria y ello requiere de un trabajo de doble vía, en donde la teoría y la evidencia se generen a partir de las necesidades de la práctica y, a su vez, se validen y prueben en la misma" (48).

En este sentido, es importante hacer evidente cómo el uso y articulación de la teoría con la práctica permiten un desarrollo disciplinar. Por ejemplo, en el caso del modelo de Callista Roy, autores como Moreno (49) plantean que "se puede relacionar con la taxonomía de enfermería y, por lo tanto, su aplicación favorece la operacionalización del proceso de enfermería" que, para este caso, permitió que la persona tuviera mayores recursos para afrontar las nuevas situaciones que vivía y, por ende, adaptarse de una mejor manera.

De igual forma, para el caso de la teoría de la incertidumbre, de acuerdo con Trejo (50), "esta teoría puede ser utilizada en una amplia gama de posibilidades ya que, tanto la persona enferma como sus familiares, se enfrentan a situaciones estresantes generadoras de incertidumbre. Es por esto que el profesional de enfermería, al conocer la teoría, puede guiar $u$ orientar a la persona para que enfoque su evaluación como una oportunidad y dirija sus estrategias a lograr la adaptación”.

Por lo tanto, es necesario articular de manera profunda "los modelos conceptuales, las teorías e indicadores empíricos como elementos generadores de sistemas de conocimiento de enfermería que se puedan utilizar como referentes para la educación, investigación y la práctica disciplinar" (51).

\section{Conclusiones}

Las personas describen sentimientos diferentes previos o posteriores a la intervención, previo a la intervención, las personas expresaron sentimientos de temor, miedo y no lograban definir con exactitud lo que significaba la cirugía. Sin embargo, luego de la intervención, sus expresiones cambiaban y veían la cirugía más como una oportunidad que como una amenaza o peligro. Además, contaban con herramientas que les posibilitaba cuidarse de una mejor forma

Los resultados de este estudio sugieren que una intervención educativa basada en un sistema conceptual-teórico-empírico muestra un cambio de conocimientos y sentimientos con relación a los cuidados postoperatorios. Sin embargo, el bajo nivel de conocimientos iniciales es preocupante, sabiendo la implicación que tiene el paciente en su recuperación posquirúrgica.

La intervención educativa sobre los cuidados postquirúrgicos permitió disminuir indirectamente 
la incertidumbre debido a que los pacientes pueden adaptarse más fácilmente a su nueva situación. Adicionalmente, el uso del lenguaje estandarizado permitió organizar intervenciones y medir los resultados de manera eficaz. Se hace evidente la importancia del papel de enfermería en el proceso de educación, dada su competencia profesional.

Este estudio presenta un ejemplo de la construcción de un sistema conceptual-teórico-empírico de conocimiento de enfermería aplicado en la práctica, articulando los conceptos de educación del modelo conceptual de adaptación de Callista Roy y el de educación de la teoría de Incertidumbre en la enfermedad de Merle Mishel, mostrando resultados positivos de una intervención enfermera con relación al nivel de conocimientos de los pacientes sobre cuidados posquirúrgicos.

\section{Conflicto de intereses}

La autora declara no tener ningún conflicto de intereses.

\section{Referencias bibliográficas}

1. Organización Mundial de la Salud (OMS). 10 datos sobre seguridad en la atención quirúrgica. [Internet]. 2008 [consultado 22 Noviembre de 2016]. Disponible en: http://www.who.int/features/ factfiles/safe_surgery/es/

2. Frederico-Avendaño C. El reto de la cirugía ambulatoria; tendencias actuales. Revista Mexicana de Cirugía [Internet]. 2013 [consultado 15 de noviembre de 2016]; 36(Suppl. 1): S167-S168. Disponible en: http://www.medigraphic.com/pdfs/rma/cma-2013/cmas131al.pdf

3. Smith CE. Perioperative nursing education: the operating room as a learning climate. Semin Perioper Nurs. [Internet]. 2001 [consultado 24 de agosto de 2016]. 10(2): 70-3. Disponible en: https://www. ncbi.nlm.nih.gov/pubmed/15129511

4. Vargas-Bermúdez Z, Fernández-Coto R. Programa de alta hospitalaria programada para preparar el egreso del paciente y familia. ev. Enfermería Actual Enfermería Actual en Costa Rica [Internet]. 2011 [consultado 4 octubre de 2016)]; 21: 1-20. Disponible en: http://www.revistas.ucr.ac.cr/index.php/ enfermeria/article/view/3658/3562

5. Herzog C. Educación al paciente y la familia en un programa de trasplante, experiencia en CLC. REV. MED. CLIN. CONDES. [Internet]. 2010 [consultado 24 de agosto de 2016]. 21(2): 293-299. Disponible en: www.sciencedirect.com/science/article/pii/S071686401070537X

6. Salazar-Maya AM. Cirugía: entre la angustia y la alegría a la vez. Aquichán [Internet]. 2011 [consultado 6 de septiembre de 2016]; 11(2): 187-198. Disponible en: http://aquichan.unisabana.edu.co/index. php/aquichan/article/view/1900/2476

7. dams HA. A perioperative education program for pediatric patients and their parents. AORN J. [Internet] 2011; [consultado 15 de Diciembre de 2016] 93(4):472-81. Disponible en: https://www. ncbi.nlm.nih.gov/pubmed/21459185

8. Kruzik N. Benefits of Preoperative Education for Adult Elective Surgery Patients. AORN Journal [Internet]. 2009[consultado 24 de Agosto de 2016] 90(3): 381-382, 385-387. Disponible en: http:// www.sciencedirect.com/science/article/pii/S0001209209005481

9. Maxwell-Downing D. The Perioperative Nursing Data Set. AORN [Internet]. 2009; [consultado 24 de Agosto de 2016] 89 (3): 600-602. Disponible en: http://www.aornjournal.org/article/S00012092(09)00187-2/references

10. Trejo F. Incertidumbre ante la enfermedad. Aplicación de la teoría para el cuidado enfermero. Enf Neurol [Internet]. 2011. [consultado 15 de Diciembre de 2016] Vol. 11, No. 1: 34-38, 2012. Disponible en: http://www.medigraphic.com/pdfs/enfneu/ene-2012/ene121g.pdf

11. Ortoleva C. An Approach to Consistent Patient Education. AORN J. [Internet]. 2010; [consultado 24 de Agosto de 2016] 92(4): 437-444. Disponible en: www.ncbi.nlm.nih.gov/pubmed/20888946

12. Lamberg E, Salanterä S, Junttila K. Evaluating Perioperative Nursing in Finland: An Initial Validation 
of Perioperative Nursing Data Set Outcomes. AORN J. [Internet]. 2013; [consultado 24 de Agosto de 2016] 98 (2): 172-185. Disponible en: https://www.ncbi.nlm.nih.gov/pubmed/23890565

13. Vargas,R.,Herrera,C.Inducción preoperatoria para pacientes y familiares en caso de cirugía mayor y su relación con la ansiedad. Rev. Enfermería Actual en Costa Rica. [Internet]. 2012; [consultado 15 de Diciembre de 2016] 22, 1---11 Disponible: https://dialnet.unirioja.es/servlet/articulo;jsessionid=CA10A6F6 01733170AF73263448E1D6ED.dialnet02? codigo $=5021227$

14. Fawcett J, Garity J. Evaluating research for evidence based nursing practice. Philadelphia: F.A. Davis Company; 2009.

15. Roy C, Heather A.. El modelo de adaptación de Callista Roy. Stanford: Editorial Appleton y Lange. $2^{\text {a }}$. ed; 1999. p. 32.

16. Mishel M. Uncertainty in illness. J. Nurs. Scholarship. [Internet].1988; [consultado Octubre de 2016] 20(4): 225-232. Disponible: http://onlinelibrary.wiley.com/doi/10.1111/j.1547-5069.1988. tb00082.x/abstract

17. Moorhead S, Johnson M, Maas M, Swanson E. (Eds.). Nursing outcomes classification (NOC) 5th ed. St. Louis, MO: Elsevier; 2013.

18. Sayin Y, Aksoy G. The nurse's role in providing information to surgical patients and family members in Turkey: a descriptive study. AORN J. [Internet]. 2012; [consultado Octubre de 2015] 95(6): 772787. Disponible en: https://www.ncbi.nlm.nih.gov/pubmed/22633384

19. Triviño-Martínez Á, Solano-Ruiz MC, Siles-González J. Application of an uncertainty model for fibromyalgia. Aten Primaria. [Internet]. 2016; 48(4): 219-25. [consultado Agosto 12 de 2016] : Disponible en: https://www.ncbi.nlm.nih.gov/pubmed/?term=Application+of+an+uncertainty+model+for+fib romyalgia.+Aten+Primaria .

20. Ferreira L, Da Silva A, Pereira M. Campos E. Acompañamiento telefónico de pacientes post prostatectomía radical: revisión sistemática. Rev. Latino-Am. Enfermagem. [Internet]. 2014; [consultado Agosto 12 de 2016] 22(2):337-45. Disponible en: www.scielo.br/pdf/rlae/v22n2/es_01041169-rlae-22-02-00337.pdf

21. Hansen B.S. Rørtveit K. Leiknes I. Morken I. Testad I. Joa I. Severinsson e. Journal of Nursing Management. [Internet]. 2012 [consultado Agosto 12 de 2016] 20, 266-277

22. Doñate $M$, Litago $C$, Monge $S$, Martínez S. Aspectos de la información preoperatoria relacionada con la ansiedad del paciente programado para cirugía. Enferm. glob. [Internet]. 2015; [consultado Agosto 12 de 2016] 14(37): 170-180. Disponible em: http://www.google.com.co/url?sa=t\&rct=j\&q=\&esrc $=$ s\&source $=$ web\&cd $=1 \& v e d=0$ ahUKEwiMzZPD3tbQAhXFYCYKHYFXB_gQFggYMAA\&url=htt p $\% 3 \mathrm{~A} \% 2 \mathrm{~F} \% 2 \mathrm{Frevistas} . u m . e s \% 2 \mathrm{Feglobal} \% 2 \mathrm{Farticle} \% 2 \mathrm{Fview} \% 2 \mathrm{~F} 185281 \&$ usg=AFQjCNEPZIkKm TIYxgKHOhjDGmNnWa9PcQ\&bvm=bv.139782543,d.eWE

23. Prata-Martineza B, Ribeiro-Silva J, Salgado-Silva V, Gomes-Neto M., Forgiarini-Júnior LA. Influencia de diferentes posiciones corporales en la capacidad vital en pacientes en el postoperatorio abdominal superior. Rev Bras Anestesiol. [Internet]. 2015; [consultado Agosto 12 de 2016] 65(3): 217-221. Disponible en: http://www.scielo.br/pdf/rba/v65n3/es_0034-7094-rba-65-03-00217.pdf

24. R Torrents, M Ricart, M Ferreiro, A López . Laura Renedo L. Lleixà M. Ferré C. Ansiedad en los cuidados: una mirada desde el modelo de Mishel. Index Enferm. 2013; 22(1-2): 60-64. Disponible en Ansiedad en los cuidados: una mirada desde el modelo de Mishel. Index Enferm. [Internet]. 2013; [consultado Agosto 12 de 2016] 22(1-2): 60-64. Disponible en http://scielo.isciii.es/scielo. php?script $=$ sci_abstract\&pid $=$ S1132-12962013001100113

25. Morales-Aguilar RS, Lastre-Amell GE, Pardo-Vásquez AC. Application of theoretical and methodological components of nursing care. Rev. cienc. cuidad. [Internet]. 2016; [consultado 15 Diciembre de 2016] 13(2): 107-120

26. Duran MM.De la teo'ría a la práctica: Necesidad de un cambio. En: Moreno ME. Muñoz L. De la teoría de enfermería a la práctica: experiencias con proyecto de gestión del cuidado. $1^{\mathrm{a}}$ ed. Chía: Universidad de La Sabana. 2016. P. 15-27

27. Acompañamiento telefónico de pacientes post prostatectomía radical: revisión sistemática Rev. Latino- 
ISSN-PRINT

$1794-9831$

E-ISSN 2322-7028

Vol. 14 No. 2

Jul - Dic 2017

Cúcuta, Colombia
Am. Enfermagem [Internet] 2014; [consultado 10 Noviembre de 2016)22(2):337-45.( Disponible en: http://www.scielo.br/pdf/rlae/v22n2/es 0104-1169-rlae-22-02-00337.pdf)

28. González-Consuegra $\mathrm{RV}^{1}$, Verdú J. Quality of life in people with venous leg ulcers: an integrative review. J Adv Nurs. [consultado 12 Agosto de 2015] 2011 May;67(5):926-44 Disponible en: https:// www.ncbi.nlm.nih.gov/pubmed/21241355

29. Woodfield J, Jamil W, Sagar P. Incidence and significance of postoperative complications occurring between discharge and 30 days: a prospective cohort study. Journal of Surgical Research. [Internet]. 2016 (consulta realizado el 12 agosto de 2016). 1. Disponible en: http://www.sciencedirect.com/ science/article/pii/S0022480416301949

30. Zimmer A. Bläuer C. Coslovsky M. Kappos L. Derfuss T. Optimizing treatment initiation: Effects of a patient education program about fingolimod treatment on knowledge, self-efficacy and patient satisfaction (internet). Multiple Sclerosis and Related Disorders. [Internet]. 2015. [consultado 3 Noviembre de 2016)] 4(5) 444-450 Disponible en: http://www.sciencedirect.com/science/article/pii/ S2211034815000814

31. Berg K. Kjellgren K. Unosson M. and Årestedt K. Postoperative recovery and its association with health-related quality of life among day surgery patients. BMC Nursing [Internet]. 2012, [Consulta realizado el 16 de agosto de 2016].11 (24). Disponible en: http://www.biomedcentral.com/1472$6955 / 11 / 24$

32. Muñoz L. El Marco lógico en la aplicación de las teorías de rango medio. En: Moreno ME. Muñoz L. De la teoría de enfermería a la práctica: experiencias con proyecto de gestión del cuidado. $1^{\mathrm{a}}$ ed. Chía: Universidad de La Sabana. 2016. P. 29-42.

33. Nanda International. Diagnósticos enfermeros definiciones y clasificación 2015-2017. Barcelona: Elsevier.2012.

34. Butcher HK, Bulechek GM, McCloskey JM, Wagner C. Nursing Interventions Classification (NIC). United States: Elsevier; 2013

35. Fawcett J, DeSanto S. The Structural of contemporary nursing Knowledge. En: Fawcett J, DeSanto S. Contemporary nursing Knowledge. Analysis an evaluation of Nursing Models and Theories. Third ed. FA Davis Company; 2013.

36. American Society of Anesthesiology . ASA Physical Status Classification System. [Internet]. [consultado 14 de diciembre de 2016] Disponible en: https://www.asahq.org/resources/clinical-information/ asa-physical-status-classification-system

37. Burns. N. Grove N. Investigación en enfermería. Desarrollo de la práctica enfermera basada en la evidencia. 5ed. Barcelona: 2012.

38. México. Comisión Nacional de Bioética. Código de Nuremberg. Normas éticas sobre experimentación en seres humanos. [Internet]. Nuremberg; Comisión Nacional de Bioética; 1947 formato [consultado 1 de Agosto de 2015] Disponible en: http://www.conbioetica-mexico.salud.gob.mx/descargas/pdf/ normatividad/normatinternacional/2.INTL._Cod_Nuremberg.pdf

39. Asociación Médica Mundial (AMM). Declaración de Helsinki de la AMM. Principios éticos para las investigaciones médicas en seres humanos. 64 Asamblea General AMM [Internet]. Fortaleza, Brasil: AMM; 2013[consultado 1 de Agosto de 2015] Disponible en: http://www.wma.net/ es/30publications/10policies/b3/

40. República de Colombia, Ministerio de Salud. Resolución No. 8430, octubre 4, por la cual se establecen las normas científicas, técnicas y administrativas para la investigación en salud. [Internet]. Bogotá D.C.: Minsalud; 1993 [consultado 1 de Agosto de 2015] Disponible en: https://www.minsalud.gov.co/ Normatividad_Nuevo/RESOLUCION\%208430\%20DE\%201993.pdf

41. Sigurdsson E, Siggeirsdottir K, Jonsson H, Gudason V, Matthiasson T, Jonsson BY. Early discharge and home intervention reduces unit costs after total hip replacement: Results of a cost analysis in a randomized study. International Journal of Health Care Finance and Economics [Internet]. 2008; [consultado 1 de Agosto de 2015] 8(3): 181-92. doi: http://dx.doi.org/10.1007/s10754-008-9036-0

42. Vonk A, Huirne JA, Brölmann HA, Emanuel MH, van Kesteren PJ, Kleiverda G, et al. Effectiveness of a multidisciplinary care program on recovery and return to work of patients after gynaecological surgery; design of a randomized controlled trial. BMC Health Serv Res [Internet]. 2012; [consultado 
24 de Agosto de 2016] 12: 29. doi:http://dx.doi.org/10.1186/1472-6963-12-29 Disponible en: www. ncbi.nlm.nih.gov/pubmed/22296950

43. Benavides $\mathrm{C}$, Prieto F, Torres $\mathrm{M}$, Buitrago G, Gaitán $\mathrm{H}$, Duarte $\mathrm{C}$, et al. Manual de práctica clínica basado en la evidencia: Controles posquirúrgicos. Rev Colomb Anestesiol [Internet]. 2015; [consultado 1 de Agosto de 2015] 43(1): 20-31. Disponible en, http://www.revcolanest.com.co/es/manual-practicaclinica-basado-evidencia/articulo/S0120334714001440/

44. Eloranta S, Katajisto J, Leino H. Orthopaedic patient education practice. Int J Orthop Trauma Nurs. [Internet]. 2016; [consultado 1 de Agosto de 2015] 21: 39-48. Disponible en https://www.ncbi.nlm. nih.gov/pubmed/26589445

45. Newhouse R, Johantgen M, Pronovost P, Johnson E. Perioperative Nurses and Patient OutcomesMortality, Complications, and Length of Stay. AORN J. [Internet]. 2005 [consultado 25 de Agosto de 2015]; 81(3): 508-509, 513-516, 518, 520-522, 525-528. Disponible en http://www.aornjournal.org/ article/S0001-2092(06)60438-9/abstract

46. Cuzco-Cabellos M, Guasch-Pomés N. Application and evalauation of care plan for patients admitted to Intensive Care Units. Enferm Intensiva. [Internet]. 2015; [consultado 24 de Agosto de 2015] 26(4): 137-43. Disponible en: https://www.ncbi.nlm.nih.gov/pubmed/26340906

47. Nogueira-Moreira RA, Áfio-Caetano J, Moreira-Barros L, Gimeniz-Galvão MT. Diagnósticos de enfermagem, fatores relacionados e de risco no pós-operatório de cirurgia bariátrica. Rev Esc Enferm USP [Internet]. 2013; formato [consultado 12 Agosto de 2015] 47(1): 168-75. Disponible en: http:// www.scielo.br/pdf/reeusp/v47n1/a21v47n1.pdf

48. Durán MM. La renovación del conocimiento y la práctica. Aquichan 2014; 14(1): 5-6.

49. Moreno-Fergusson M, Alvarado-García A. Aplicación del Modelo de Adaptación de Callista Roy en Latinoamérica: revisión de la literatura. Aquichan [Internet]. 2009 [consultado 2 de agosto de 2016]; 9(1): 62-72. Disponible en: http://aquichan.unisabana.edu.co/index.php/aquichan/article/view/170/1671

50. Trejo-Martínez F. Incertidumbre ante la enfermedad. Aplicación de la teoría para el cuidado enfermero. Enf Neurol. (Mex) [Internet]. 2012; [consultado 2 de agosto de 2016] 11(1): 34-38. Disponible en: http://www.medigraphic.com/pdfs/enfneu/ene-2012/ene121g.pdf

51. Durán M. Contemporary Nursing Knowledge: Analysis and Evaluation of Nursing Models and Theories. Aquichan, Norteamérica [Internet]. 2009 [consultado 2 de octubre de 2016]; 5 (19). Disponible en: http://aquichan.unisabana.edu.co/index.php/aquichan/article/view/73/151. 